03,13

\title{
Фотоэмиссионные исследования электронной структуры GaN, выращенного методом молекулярно-пучковой эпитаксии с плазменной активацией азота
}

\author{
(C) С.Н. Тимошнев ${ }^{1}$, А.М. Мизеров ${ }^{1}$, Г.В. Бенеманская ${ }^{2}$, С.А. Кукушкин ${ }^{3}$, А.Д. Буравлев ${ }^{1}$ \\ ${ }^{1}$ Санкт-Петербургский национальный исследовательский Академический университет РАН, \\ Санкт-Петербург, Россия \\ ${ }^{2}$ Физико-технический институт им. А.Ф. Иофффе РАН, \\ Санкт-Петербург, Россия \\ ${ }^{3}$ Санкт-Петербургский национальный исследовательский университет информационных технологий, механики и оптики, \\ Санкт-Петербург, Россия \\ E-mail: timoshnev@mail.ru
}

Поступила в Редакцию 16 июля 2019 г.

В окончательной редакции 16 июля 2019 г.

Принята к публикации 25 июля 2019 г.

\begin{abstract}
Представлены результаты экспериментальных исследований электронных и фотоэмиссионных свойств эпитаксиального слоя $\mathrm{GaN}$, выращенного методом молекулярно-пучковой эпитаксии с плазменной активацией азота на подложке $\mathrm{SiC} / \mathrm{Si}(111)$. Электронная структура поверхности $\mathrm{GaN}$ и ультратонкого интерфейса $\mathrm{Li} / \mathrm{GaN}$ была впервые исследована in situ в условиях сверхвысокого вакуума при различных покрытиях Li. Эксперименты проводились с использованием фотоэлектронной спектроскопии при синхротронном излучении в диапазоне энергий фотонов $75-850 \mathrm{eV}$. Спектры фотоэмиссии в области валентной зоны и поверхностных состояний и спектры фотоэмиссии из остовных уровней $\mathrm{N} 1 s, \mathrm{Ga} 3 d$, Li $2 s$ были изучены при различных субмонослойных покрытиях Li. Установлено, что адсорбция Li вызывает существенные изменения в общем виде спектров, вызванные переносом заряда между слоем Li и нижними слоями $\mathrm{N}$ и Ga. Установлено, что поверхность $\mathrm{GaN}$ имеет преимущественно $\mathrm{N}$-полярность. Показан полупроводниковый характер интерфейса $\mathrm{Li} / \mathrm{GaN}$.
\end{abstract}

Ключевые слова: нитрид галлия, структура валентной зоны, фотоэлектронная спектроскопия.

DOI: $10.21883 /$ FTT.2019.12.48536.15ks

\section{1. Введение}

Нитриды III группы представляют большой интерес благодаря их уникальным электрическим и оптическим свойствам. III-нитриды являются наиболее перспективными материалами для новых применений в мощных высокочастотных электронных устройствах, а также в оптоэлектронных устройствах, работающих в широком спектральном диапазоне от инфракрасного до ультрафиолетового [1,2].

Высокотехнологичные приложения в наноэлектронике требуют всестороннего знания структурных и электронных свойств объема и поверхности GaN. Изменения характеристик поверхности могут быть вызваны взаимодействием с адсорбированными атомами. Поэтому исследования основополагающих принципов формирования интерфейсов являются обязательными для базового понимания поведения устройств. Электронная структура поверхности $\mathrm{GaN}$ изучалась во многих аспектах [3-9], но границы раздела металл/GaN все еще недостаточно изучены, и поэтому они определяют необходимость дополнительных исследований поверхностных состояний, формирования границ раздела, изгиба зон. Эти аспекты могут иметь решающее значение для наноструктур, где граничные условия играют ключевую роль.
Наиболее полную информацию об электронной структуре дают результаты фотоэмиссионных исследований с использованием фотоэлектронной спектроскопии (ФЭС). Недавно были исследованы электронные и фотоэмиссионные свойства границ раздела $\mathrm{Ru} / \mathrm{GaN}, \mathrm{Cs} / \mathrm{GaN}$ и $\mathrm{Ba} / \mathrm{GaN}$ [10-15]. Электронная структура интерфейса $\mathrm{Li} / \mathrm{GaN}$ изучена недостаточно.

B данной работе мы представляем подробные исследования значительного изменения электронных и фотоэмиссионных свойств из-за влияния адсорбции Li на поверхность $\mathrm{GaN}(0001)$ в диапазоне субмонослойных покрытий. Показано, что спектры валентной зоны и поверхностных состояний и спектры остовных уровней $\mathrm{Ga} 3 d$ и $\mathrm{N} 1 s$ существенно модифицируются за счет адсорбции Li.

\section{2. Эксперимент}

Эпитаксиальные слои $\mathrm{GaN}$ были выращены методом молекулярно-пучковой эпитаксии с плазменной активацией азота на установке Veeco GEN200. Для активации азота использовался высокочастотный (13.56 Mhz) плазменный источник Riber RFN 50/63. Температура подложки контролировалась in situ инфракрасным пирометром. Для выращивания нелегированных слоев $\mathrm{GaN}$ 
использовались гибридные подложки $\mathrm{SiC}$, полученные путем замещения атомов на подложках $\mathrm{Si}(111)$ [16]. Термическая очистка подложки $\mathrm{SiC} / \mathrm{Si}(111)$ осуществлялась in situ во время отжига при температуре $T_{S}=850^{\circ} \mathrm{C}$ в течение $30 \mathrm{~min}$. После этого подложка была подвергнута процессу нитридизации при температуре $T_{S}=850^{\circ} \mathrm{C}$ в течение $60 \mathrm{~min}$ с целью формирования нитридного поверхностного слоя. Условия эпитаксиального роста были использованы как в работе [17]. Рост $\mathrm{GaN}$ осуществлялся в два этапа. На первом этапе при температуре подложки $T_{S}=650^{\circ} \mathrm{C}$ и равных потоках галлия и активированного азота $F_{\mathrm{Ga}}=F_{\mathrm{N}}=0.1 \mu \mathrm{m} / \mathrm{h}$ формировался зародышевый слой $\mathrm{GaN}$ толщиной $200 \mathrm{~nm}$. Дальнейший рост основного слоя осуществлялся при температуре подложки $T_{S}=730^{\circ} \mathrm{C}$ и соотношении потоков $\mathrm{Ga}$ и активированного азота $F_{\mathrm{Ga}} / F_{\mathrm{N}}=6$ при неизменном потоке азота. Толщина образца $\mathrm{GaN}$ составляла $\sim 870 \mathrm{~nm}$.

Фотоэмиссионные исследования проводились на российско-германской линии излучения синхротрона BESSY II (Берлин, Германия) с помощью ФЭС при синхротронном излучении с энергиями фотонов в диапазоне 75-850 eV. Эксперименты проводились in situ в сверхвысоком вакууме $5 \cdot 10^{-10}$ Torr при комнатной температуре. Регистрировались спектры нормальной фотоэмиссии электронов из валентной зоны и спектры остовных уровней $\mathrm{Ga} 3 d, \mathrm{~N} 1 s$ и Li $2 s$. Общее энер-

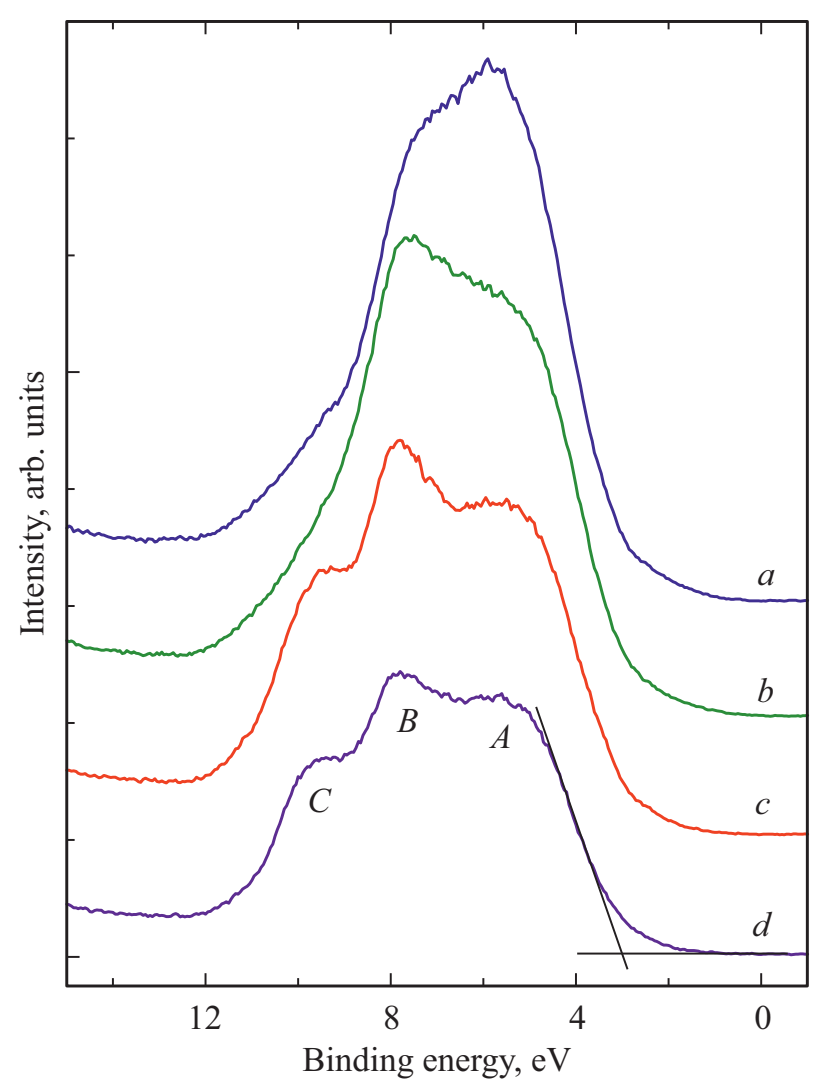

Рис. 1. Фотоэмиссионные спектры в области валентной зоны для чистой поверхности $\mathrm{GaN}$ при различных энергиях возбуждения $h v$.

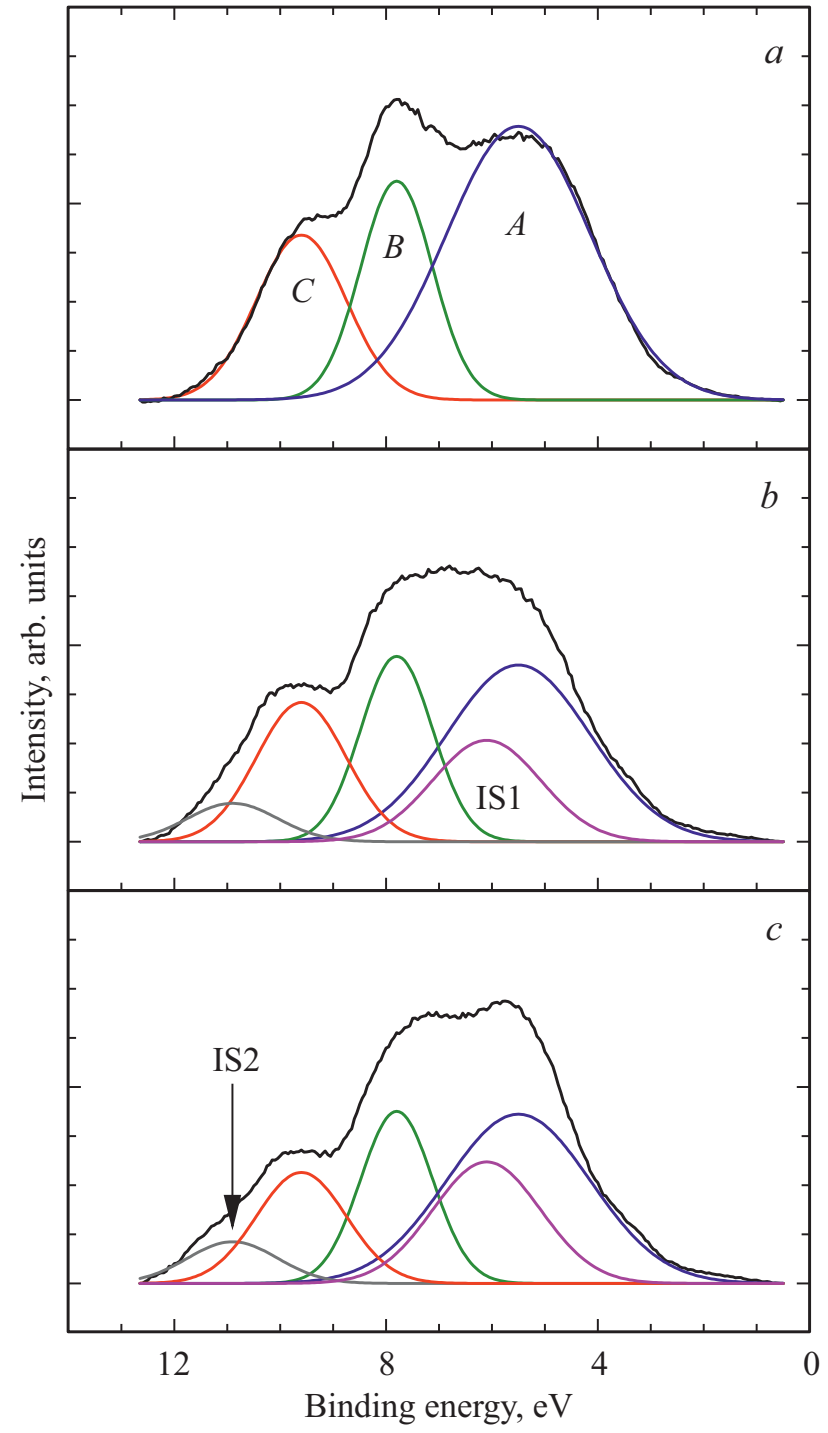

Рис. 2. Фотоэмиссионные спектры в области валентной зоны для чистой поверхности $\mathrm{GaN}$ и интерфейса $\mathrm{Li} / \mathrm{GaN}$. Энергия возбуждения $h v=130 \mathrm{eV}$.

гетическое разрешение анализатора и монохроматора составляло $50 \mathrm{meV}$.

Перед фотоэмиссионными исследованиями образец подвергался отжигу in situ при температуре $950 \mathrm{~K}$. Атомарно-чистый Li напылялся пошагово на чистую поверхность образца из стандартного источника. Отметим, что за один монослой (ML) Li принимается концентрация $\sim 1.0 \cdot 10^{15}$ atom $/ \mathrm{cm}^{2}$, при которой формируется укомплектованный слой атомов Li. Покрытие атомов лития определялось по ослаблению остовных уровней Ga $3 d$ и $\mathrm{N} 1 s$.

\section{3. Результаты и обсуждение}

На рис. 1 представлены фотоэмиссионные спектры в области валентной зоны $\mathrm{GaN}$ при различных энергиях возбуждения: $a-110 \mathrm{eV}, b-120 \mathrm{eV}, c-130 \mathrm{eV}$, 


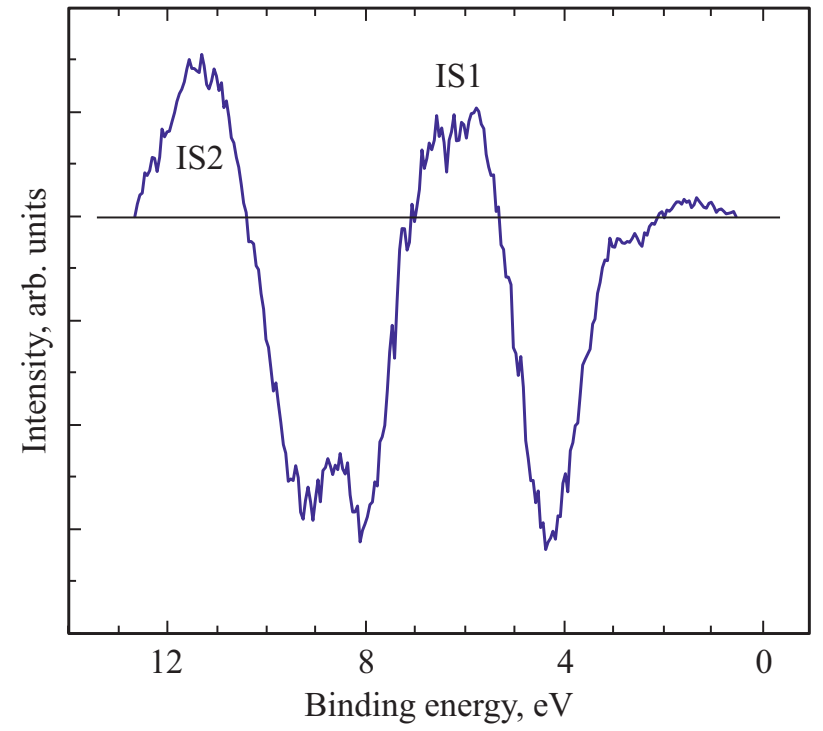

Рис. 3. Разностный спектр для интерфейса Li/GaN при Li покрытии $1.0 \mathrm{ML}$ и чистого образца GaN. Энергия возбуждения $h v=130 \mathrm{eV}$.

$d-150 \mathrm{eV}$. В области валентной зоны спектр исходного образца $\mathrm{GaN}$ представляет собой структурированную полосу фотоэмиссии шириной $\sim 9 \mathrm{eV}$. Форма спектров значительно зависит от энергии возбуждения. Полученные нами спектры находятся в хорошем согласии с теоретическими и экспериментальными данными [3,9]. В спектрах фотоэмиссии наблюдаются три особенности. Пики А и С связаны с гибридизированными состояниями $\mathrm{Ga} 4 p-\mathrm{N} 2 p$ и $\mathrm{Ga} 4 s-\mathrm{N} 2 p$, соответственно с доминантным характером $\mathrm{N} 2 p$, тогда как пик $\mathrm{B}$ приписывается смешанным орбиталям или поверхностным адсорбатам [3]. Также было определено положение вершины валентной зоны (VBM) на поверхности $E_{\mathrm{VBM}}$ путем линейной аппроксимации низкоэнергетического края спектра. Оказывается, что VBM лежит на $\sim 3.0 \mathrm{eV}$ ниже уровня Ферми на поверхности для образца $\mathrm{GaN}$. Принимая во внимание ширину запрещенной зоны $\left(E_{g}\right)$ $\mathrm{GaN} \sim 3.4 \mathrm{eV}$ и положение VBM, можно утверждать, что непреднамеренно легированный образец $\mathrm{GaN}$ демонстрирует $n$-проводимость.

Установлено, что адсорбция $\mathrm{Li}$ на поверхность $\mathrm{GaN}$ вызывает существенное изменение во всей области валентной зоны. На рис. 2 представлены фотоэмиссионные спектры в области валентной зоны для чистой поверхности $\mathrm{GaN}(a)$ и для интерфейса $\mathrm{Li} / \mathrm{GaN}$ при различных субмонослойных покрытиях $\mathrm{Li}: b-0.5 \mathrm{ML}, c-1.0 \mathrm{ML}$. Разложение спектров осуществляется путем разделения отдельных пиков с использованием асимметричных гауссовских функций. Четко наблюдаются три особенности при энергиях связи $\sim 5.7 \mathrm{eV}(\mathrm{A}), \sim 7.8 \mathrm{eV}(\mathrm{B})$, $\sim 9.5 \mathrm{eV}$ (С). При адсорбции $\mathrm{Li}$ на поверхность GaN интенсивность пиков А, В и С уменьшается. Также по мере увеличения литиевого покрытия возникают новые индуцированные поверхностные состояния IS1 и IS2 при энергиях связи $6.1 \mathrm{eV}$ и $10.9 \mathrm{eV}$, соответственно. Интерфейс $\mathrm{Li} / \mathrm{GaN}$ характеризуется как полупроводниковый.

На рис. 3 представлен спектр, соответствующий разности интенсивностей фотоэмиссии от поверхности $\mathrm{GaN}$, покрытой $\mathrm{Li}$ (рис. 2,c), и от чистой поверхности $\mathrm{GaN}$ (рис. 2,a). Разностный спектр позволяет нам визуализировать основное изменение электронной структуры при адсорбции Li и продемонстрировать уменьшение поверхностных состояний $\mathrm{A}, \mathrm{B}$ и С, а также образование зон IS1 и IS2, индуцированных адсорбцией Li. Следует отметить, что наши измерения ФЭС не показали наличия аккумуляционного слоя на чистой поверхности $\mathrm{GaN}$, а также на поверхности $\mathrm{GaN}$, покрытой Li.
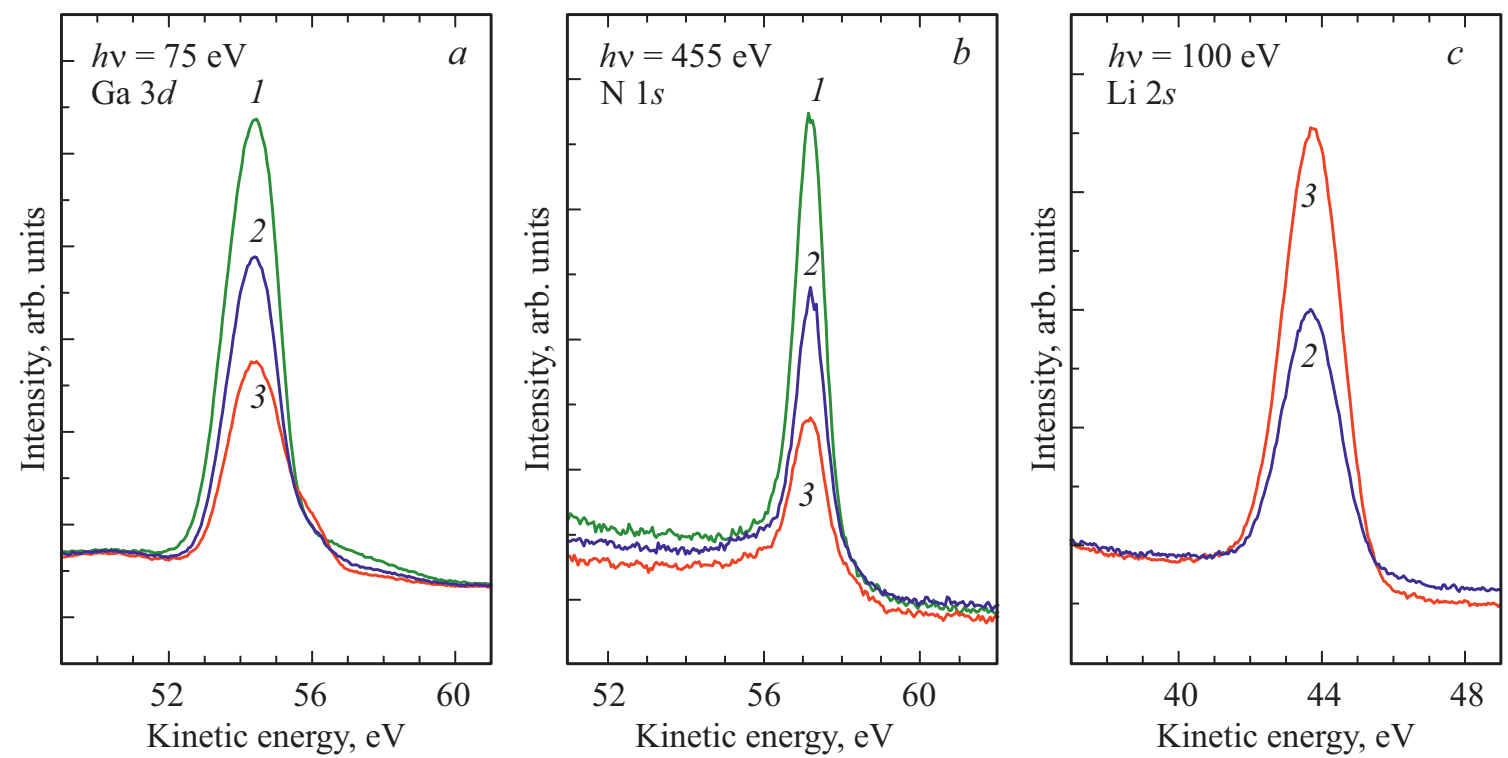

Рис. 4. Спектры нормальной фотоэмиссии из остовных уровней Ga $3 d(a), \mathrm{N} 1 s(b)$ и $\mathrm{Li} 2 s(c)$ для чистой поверхности GaN $(1)$ и для интерфейса $\mathrm{Li} / \mathrm{GaN}$ при субмонослойных покрытиях Li $0.5 \mathrm{ML}$ (2) и $1.0 \mathrm{ML}(3)$ при различных энергиях возбуждения $h v$. 
На рис. 4, $a$ представлены спектры фотоэмиссии из остовного уровня $\mathrm{Ga} 3 d$ для чистой поверхности $\mathrm{GaN}$ и интерфейса $\mathrm{Li} / \mathrm{GaN}$ при энергии возбуждения $75 \mathrm{eV}$. Спектр фотоэмиссии из остовного уровня $\mathrm{Ga} 3 d$ при такой энергии возбуждения соответствует поверхностной фотоэмиссии, так как кинетическая энергия вылетевших фотоэлектронов близка к минимуму на универсальной кривой зависимости длины свободного пробега электронов от энергии. На рис. $4, b$ приведены спектры фотоэмиссии из остовного уровня $\mathrm{N} 1 s$ для чистой поверхности $\mathrm{GaN}$ и интерфейса $\mathrm{Li} / \mathrm{GaN}$ при энергии возбуждения $455 \mathrm{eV}$. Энергия возбуждения была подобрана таким образом, чтобы кинетические энергии фотоэлектронов из остовных уровней $\mathrm{N} 1 s$ и $\mathrm{Ga} 3 d$ были приблизительно равны. Поэтому спектр фотоэмиссии из остовного уровня $\mathrm{N} 1 s$ при такой энергии возбуждения тоже соответствует поверхностной фотоэмиссии. Исследование спектров фотоэмиссии из Ga $3 d, \mathrm{~N} 1 s$ и $\mathrm{Li} 2 s$ свидетельствует о том, что форма остовных уровней при адсорбции $\mathrm{Li}$ изменяется незначительно. Происходит уменьшение интенсивности пиков $\mathrm{Ga} 3 d$ и $\mathrm{N} 1 s$ в 2 и 3 раза, соответственно, при покрытии Li $1.0 \mathrm{ML}$, и наблюдается рост интенсивности фотоэмиссии из остовного уровня Li $2 s$. Поскольку интенсивность фотоэмиссии остовного уровня $\mathrm{N} 1 s$ уменьшилась больше, чем $\mathrm{Ga} 3 d$, мы полагаем, что образец $\mathrm{GaN}$ имеет преимущественно $\mathrm{N}$-полярную поверхность. Эти данные подтверждаются исследованиями полярности других образцов, выращенных при аналогичных условиях [17].

\section{4. Заключение}

Эпитаксиальный слой $\mathrm{GaN}$ был выращен методом молекулярно-пучковой эпитаксии с плазменной активацией азота на гибридной подложке $\mathrm{SiC} / \mathrm{Si}(111)$. Электронная структура поверхности $\mathrm{GaN}$ и ультратонкой границы раздела $\mathrm{Li} / \mathrm{GaN}$ была впервые исследована при различных субмонослойных покрытиях Li. Эволюция спектров поверхностных состояний и спектров остовных уровней с ростом покрытия Li демонстрирует адсорбционную активность N- и Ga-оборванных связей на поверхности $\mathrm{GaN}$. Образец $\mathrm{GaN}$ имеет преимущественно $\mathrm{N}$-полярную поверхность. Для чистой поверхности $\mathrm{GaN}$ найдены собственные поверхностные состояния А, В и С при энергиях связи $\sim 5.7 \mathrm{eV}, \sim 7.8 \mathrm{eV}, \sim 9.5 \mathrm{eV}$, соответственно. Адсорбция лития приводит к возникновению новых индуцированных поверхностных состояний IS1 и IS2 при энергиях связи $6.1 \mathrm{eV}$ и $10.9 \mathrm{eV}$, соответственно. Обнаружено незначительное подавление состояний A, B и С за счет адсорбции Li. Данные могут быть объяснены вызванным адсорбцией Li насыщением оборванных связей $\mathrm{N}$ и $\mathrm{Ga}$, что приводит как к подавлению собственных поверхностных состояний, так и к полупроводниковой природе границы раздела $\mathrm{Li} / \mathrm{GaN}$.

\section{Благодарности}

Авторы выражают благодарность Helmholtz-Zentrum Berlin и русско-немецкой линии синхротрона BESSY II за предоставленные возможности для проведения экспериментов и за помощь во время экспериментов.

\section{Финансирование работы}

Ростовые эксперименты проводились в рамках выполнения государственного задания Министерства образования и науки Российской Федерации № 16.9789.2017/БЧ. Фотоэмиссионные исследования образцов проводились в рамках общего соглашения о научно-исследовательской деятельности между Сколтех и Санкт-Петербургским национальным исследовательским Академическим университетом РАН (№ 3663-МРА, проект 4).

\section{Конфликт интересов}

Авторы заявляют, что у них нет конфликта интересов.

\section{Список литературы}

[1] A. Chakraborty, B.A. Haskell, S. Keller, J.S. Speck, S.P. DenBaars, S. Nakamura, U.K. Mishra. Jpn. J. Appl. Phys. 44, L173 (2005).

[2] S.P. DenBaars, D. Feezell, K. Kelchner, S. Pimputkar, C.C. Pan, C.C. Yen, S. Tanaka, Y. Zhao, N. Pfaff, R. Farrell, M. Iza. Acta Mater. 61, 945 (2013).

[3] W.R.L. Lambrecht, B. Segall, S. Strite, G. Martin, A. Agarwal, H. Morkoç, A. Rockett. Phys. Rev. B. 50, 14155 (1994).

[4] T. Strasser, C. Solterbeck, F. Starrost, W. Schattke. Phys. Rev. B. 60, 11577 (1999).

[5] G.V. Benemanskaya, M.N. Lapushkin, S.N. Timoshnev. Surf. Sci. 603, 2474 (2009).

[6] D. Yujie, C. Benkang, W. Xiaohui, Z. Junju, L. Biao, W. Meishan. Appl. Surf. Sci. 258, 7425 (2012).

[7] M. Lozac, S. Ueda, S. Liu, H. Yoshikawa, S. Liwen, X. Wang, B. Shen, K. Sakoda, K. Kobayashi, M. Sumiya. Sci. Technol. Adv. Mater. 14, 015007 (2013).

[8] A. Eisenhardt, S. Krischok, M. Himmerlich. Appl. Phys. Lett. 102, 231602 (2013).

[9] D. Skuridina, D.V. Dinh, B. Lacroix, P. Ruterana, M. Hoffmann, Z. Sitar, M. Pristovsek, M. Kneissl, P. Vogt. J. Appl. Phys. 114, 173503 (2013).

[10] R. Wasielewski, M. Grodzicki, J. Sito, K. Lament, P. Mazur, A. Ciszewski. Acta Phys. Pol. A. 132, 354 (2017).

[11] C.I. Wu, A. Kahn. Appl. Surf. Sci. 162-163, 250 (2000).

[12] F. Machuca, Y. Sun, Z. Liu, K. Ioakeimidi, P. Pianetta, R.F.W. Pease. J. Vac. Sci. Technol. B. 18, 3042 (2000).

[13] T.U. Kampen, M. Eyckeler, W. Moench. Appl. Surf. Sci. 123124, 28 (1998).

[14] G.V. Benemanskaya, S.A. Kukushkin, P.A. Dementev, M.N. Lapushkin, S.N. Timoshnev, D.V. Smirnov. Solid State Commun. 271, 6 (2018).

[15] G.V. Benemanskaya, S.N. Timoshnev, S.V. Ivanov, G.E. FrankKamenetskaya, D.E. Marchenko, G.N. Iluridze. JETP. 118, 600 (2014).

[16] S.A. Kukushkin, A.V. Osipov. J. Appl. Phys. 113, 0249091 (2013).

[17] S.A. Kukushkin, A.M. Mizerov, A.V. Osipov, A.V. Redkov, S.N. Timoshnev. Thin Solid Films. 646, 158 (2018).

Редактор К.В. Емщев 